\title{
EXPLORANDO VIRTUDES EN UN INSTITUTO PÚBLICO MEXICANO DE EDUCACIÓN SECUNDARIA
}

\section{EXPLORING VIRTUES IN A MEXICAN PUBLIC SECONDARY SCHOOL}

\author{
Apolinar Varela, Concepción Naval, \\ Aurora Bernal y Ángel Sobrino
}

Dr. en Ciencias de la Educación. Investigador post-doc, Instituto Cultura y Sociedad, Facultad de Educación y Psicología, Universidad de Navarra.

agrana@unav.es

Catedrática en Teoría e Historia de la Educación. IP del grupo de investigación "Educación, ciudadanía y carácter". Decana de la Facultad de Educación y Psicología, Universidad de Navarra. cnaval@unav.es

Dra. en Ciencias de la Educación. Profesora titular en Teoría e Historia de la Educación. Vicedecana de Ordenación Académica de la Facultad de Educación y Psicología, Universidad de Navarra. abernal@unav.es

Dr. en Ciencias de la Educación. Profesor titular de "Métodos de Investigación y Diagnóstico en Educación", Universidad de Navarra. Vicedecano de investigación en la Facultad de Educación y Psicología, Universidad de Navarra.

asobrino@unav.es 


\section{RESUMEN}

Desde tiempos inmemoriales, el desarrollo social y moral de la persona es un tema que preocupa a todo tipo corrientes filosóficas, pedagógicas, sociológicas o psicológicas. En esta investigación, mediante un estudio de casos cualitativo, nos proponemos averiguar cómo se expresan las virtudes en el contexto de un instituto público mexicano de Educación Secundaria, a través de tres docentes de Formación cívica y ética. Constatamos la importancia de la reflexión del docente sobre su compromiso moral, ético y pedagógico con el contexto sociocultural que le rodea y cómo esto afecta a la práctica de determinadas virtudes.

Palabras clave: educación secundaria, virtudes, educación del carácter, México.

\section{ABSTRACT}

From time immemorial, the social and moral development of the individual has been a subject of interest for all philosophical, pedagogical, sociological and psychological schools of thought. In this research, using qualitative case study, we intend to establish how virtues are expressed in the context of a Mexican public secondary school through contact with three teachers of Civics and Ethics. We are aware of the importance of teachers' reflections on the moral, ethical and pedagogical commitment to their socio-cultural environment and how this affects the practice of certain virtues.

Key words: secondary Schools, virtues, character education, México.

\section{INTRODUCCIÓN}

Desde tiempos inmemoriales, el desarrollo social y moral de la persona es un tema de interés para todo tipo de corrientes filosóficas, pedagógicas, sociológicas o psicológicas. En el proceso formativo de una persona, la escuela - y especialmente los docentes- poseen un cierto protagonismo durante la infancia y la adolescencia, pudiendo impactar en el desarrollo moral del alumnado durante toda su vida, «aunque 
solamente nos demos cuenta de su contribución a nuestro desarrollo en retrospectiva» (Sanderse, 2013, p. 31).

Desde hace tres décadas, el interés por la educación moral y cívica ha ido aumentando a nivel internacional (Altarejos y Naval, 2000; Naval, Fuentes, y Quintanilla, 2017). No obstante, parece todavía de manera insuficiente en el contexto mexicano (Carbajal, 2009; Lozano, 2015; Yurén, Saenger, y Rojas, 2014). Ante este escenario, nos proponemos averiguar cómo se expresan las virtudes en el contexto de un instituto público emergente (Milner, 2015) mexicano de Educación Secundaria, a través de tres docentes de formación cívica y ética. Conocedores de que la educación moral y cívica ofrece distintas perspectivas - se comentarán en el siguiente apartado-, nuestra aproximación responde al enfoque denominado «educación del carácter».

\section{PERSPECTIVAS EN TORNO A LA EDUCACIÓN MORAL Y ÉTICA}

A lo largo del tiempo, han surgido distintas perspectivas que abordan el tema de la educación moral y social. Una de las clásicas dentro de las aulas es la clarificación de valores, cuyo objetivo es que los estudiantes aprendan a realizar un proceso de reflexión, que ayude a la adquisición de una conciencia moral crítica y responsable (Carrillo, 1992). Este enfoque busca el autodescubrimiento de la persona a través de una serie de ejercicios estructurados que ayudan a que cada persona pueda discernir qué debe o no debe hacer, sin que el docente ejerza como un agente moral adoctrinador.

Otro de los enfoques que se aproxima a la educación ética y moral se origina desde el campo de la psicología, centrado en el desarrollo moral a partir del desarrollo cognitivo de las personas. En este sentido Piaget con su obra El criterio moral en el niño (1984) y su enfoque constructivista (Devries, 1999) con sus cuatro estadíos de desarrollo cognitivo en torno a la regla en los juegos infantiles, sirvió de base para la perspectiva cognitivo-formalista de Kohlberg (Bolívar, 1987). En ésta se busca que la persona sea autónoma para razonar distintos juicios morales desde un punto de vista imparcial. 
Kohlberg aumenta los estadíos del desarrollo moral de cuatro a seis, y apunta que el crecimiento moral de una persona de un nivel a otro se produce como resultado del conflicto cognitivo interno. Para alcanzar esta madurez sugiere los famosos dilemas morales en los que la persona deberá optar por un determinado rol ante una situación concreta y lo que denomina como «Just community schools» (Bolívar, 1987). En éstas, la participación de los escolares en temas de organización escolar y de carácter moral es fundamental para estimular el desarrollo moral.

El posicionamiento de Kohlberg fue criticado por Gilligan (Gordi1lo, 1987), quien considera que carece de la perspectiva de la mujer y de su crecimiento a la hora de alcanzar las distintas fases de desarrollo (Gordillo, 1987). Esta preocupación por proporcionarle un nuevo enfoque a la educación moral desde una visión más próxima a la feminidad, tendrá un lugar en la ética del cuidado propuesta por Noodings (n.d., 1984, 2008), que fue su principal exponente. Desde este enfoque el cuidado hacia los demás se entiende como la mejor base para la educación moral, partiendo de que «los docentes muestran a los alumnos cómo cuidar, involucrándoles en el diálogo sobre la vida moral, supervisando su práctica en el cuidado y confirmándoles cómo desarrollar lo mejor de sí mismos» (Noddings, n.d).

Otra perspectiva que también se centra en el desarrollo moral y cívico de la persona es la educación del carácter. Este enfoque se remonta a la Grecia clásica y posee en Aristóteles a su mayor exponente (Bernal, 1998). Esta educación se centra en la búsqueda del florecimiento de las virtudes individuales y cívicas a través de su práctica diaria, la observación de modelos ejemplares y la orientación a la reflexión crítica (Arthur y Kristjánsson, 2015). En la actualidad ha resurgido con fuerza a nivel internacional desde distintas aproximaciones que van desde lo psicológico a lo cívico (Bernal, González-Torres y Naval, 2015). La educación del carácter es, junto con la ética del cuidado, la que mayor importancia le otorga al role-modeling (Sanderse, 2013). Esto es así a pesar de que los docentes pueden mostrar rasgos poco admirables y que pueden no ser efectivos (Sanderse, 2013), ya que factores como la identidad, la raza, el género o la religión afectan de manera clara a la hora de seguir un determinado modelo (Martino, 2009; Moulin-Stozek y Schirr, 2017). 
En este sentido, Kristjánsson (2006) indica que el docente es modelo por ser profesor y es un mentor moral que guía en la transición de la infancia a la juventud desde la experiencia. Así, el docente debe ser capaz de ayudar a florecer las virtudes personales en los escolares atendiendo al método, el estilo, las formas o la personalidad del docente (Fallona, 2000; Fenstermacher, 1992; Fenstermacher, Osguthorpe, y Sanger, 2009; Richardson y Fallona, 2001; Willemse, Lunenberg, y Korthagen, 2008), así como a su conocimiento y sabiduría prácticas (Biesta, 2014). En definitiva, teniendo en cuenta todos los elementos que se manifiestan en el currículo oculto de las escuelas, las aulas y los docentes (Jackson, 1991). «La importancia de su rol no se basa, exclusivamente, en lo que enseñan directamente, sino también en lo que modelan en la práctica como modelos ejemplares» (Arthur, Kristjánsson, Harrison, Sanderse, y Wright, 2017, p. 5).

\section{METODOLOGÍA}

En la investigación hemos empleado la metodología cualitativa del estudio de casos (Simons, 2011), pues permite aproximarnos al estudio de las virtudes de una manera holística, explorando las distintas interacciones sociales, más o menos simbólicas, que expresan los agentes sociales, en función de su rol y propósitos personales, en un determinado contexto y que influyen en el proceso social del carácter (Moulin-Stozek, 2018). Este posicionamiento nos permite observar qué tipo de virtudes manifiestan tres docentes y cómo tratan de proyectarlas en su día a día.

Para la recogida de datos hemos utilizado la entrevista semiestructurada. Estas entrevistas han sido grabadas y transcritas; su duración oscila entre los 70 y 90 minutos, cada una. Para completar la recogida de datos mantuvimos conversaciones, más o menos informales, que denominamos «entrevistas etnográficas» (Spradley, 1979). La realización de las entrevistas y el acceso a las aulas fueron negociados con cada uno de ellos a través de acuerdos verbales (Stake, 1998), garantizando la confidencialidad y el anonimato de sus declaraciones. Además, contamos con el consentimiento informado por parte de la administración educativa, la dirección de centro y los tutores legales de los menores. 
Las entrevistas semiestructuradas estaban organizadas en distintos bloques de preguntas, en las que se les cuestionaba sobre algunas virtudes (coraje, honestidad...), cómo trataban de enseñar los contenidos, los modelos a seguir, los tipos de rutinas en el aula, los contenidos, etcétera.

Otro elemento ha sido la observación participante (Angrosino, 2012) en las clases y en el propio instituto. Una gran parte de las observaciones realizadas han sido grabadas en vídeo, lo que ofrece la oportunidad de visualizar y analizar las sesiones a posteriori (Andersson y Sørvik, 2013). En nuestro caso, hemos empleado observaciones descriptivas y focalizadas (Spradley, 1980). Otras sesiones han sido grabadas en audio y acompañadas por notas de campo. Durante la estancia en el campo observamos algunas de las clases impartidas por los docentes, otras actividades (honores a la bandera, etcétera) y diferentes espacios escolares de interacción. En este caso hemos estado presentes en el centro durante 3 semanas todos los días, desde las 7 de la mañana hasta bien entrada la tarde. Completamos un total de 19 sesiones impartidas por estos docentes, siendo las clases de Formación de cívica y Ética las que ocupan un mayor volumen (14), y menos la asignatura estatal (4). La variedad de las fuentes, la triangulación metodológica (Denzin, 1989) y el tratamiento de los mismos, garantiza una mayor fiabilidad y credibilidad en el estudio (Goetz y LeCompte, 1988).

\section{ANÁLISIS DE DATOS}

Para el análisis de los datos nos hemos apoyado en un razonamiento deductivo-inductivo. Este tipo de aproximación a los datos se orienta a buscar una mayor validez externa del estudio. Una de las características de la investigación cualitativa es la simultaneidad entre la recogida de datos y el análisis. De este modo, en una primera fase del análisis hemos empleado la interpretación directa (Stake, 1998) durante la recogida de datos. Posteriormente hemos utilizado, para la elaboración de la codificación provisional (Miles, Huberman, y Saldaña, 2014), diferente literatura, principalmente de inspiración aristotélica (Arthur et al., 2017; Campell, 2013; Fallona, 2000; Fenstermacher, 1992; Fenstermacher et al., 2009; Klaassen, 2010; Kristjánsson, 2006; Martino, 2009; 
Richardson y Fallona, 2001; The Jubilee Centre for Character y Virtues, 2017) y complementada con los temas emergentes en los datos. Esta manera de proceder facilita aplicar el razonamiento inductivo-deductivo o viceversa, y realizar el análisis cruzado de los datos (Taylor y Bogdan, 1992).

\section{EL ESCENARIO ESCOLAR}

El escenario en el que se desarrolla el estudio es un instituto público urbano emergente (Milner, 2015) de Educación Secundaria de México, situado en el interior del país. Se ubica en una zona rodeada de barrios deprimidos económicamente y con problemas sociales graves. De estas áreas procede más del $70 \%$ de la población que acude a este colegio, por lo que podemos hablar de un estrato socioeconómico bajo. La población escolar total es de 1.598 escolares organizados en dos turnos, matutino y vespertino; en el primer turno estaban censados 857 escolares y en el vespertino 741 estudiantes. Cada turno se divide en 3 cursos académicos con 6 grupos por curso. Cada grupo está formado por 48 alumnos. El acceso al centro fue negociado con las autoridades estatales competentes en materia educativa.

Durante esta investigación centramos la atención sobre tres docentes de Formación cívica y Ética. Nuestros informantes han sido seleccionados gracias al criterio de selección primaria (Morse como se citó en Flick, 2004, p. 83). En nuestro estudio contamos con la participación de Gabriel ${ }^{1}$, licenciado en Ciencias Sociales que imparte docencia en el mismo centro desde hace 40 años, y cuya jubilación en el momento de la investigación estaba próxima, por lo que la experiencia y conocimiento sobre el contexto social de la escuela es amplísimo. Gabriel imparte las materias de Formación cívica y Ética, Tutoría y Geografía, en el turno matutino y vespertino; además es el encargado de ayudar a gestionar la sociedad del alumnado, participa activamente en los proyectos en la escuela y es el encargado de evaluar. Otro docente es Pedro con una vasta experiencia sobre el centro y la problemática escolar. Él

1 Los nombres han sido alterados para mantener el anonimato y la confidencialidad de nuestros informantes. 
es licenciado en Psicología con especialización en educación, lleva trabajando en el instituto 37 años, pero con anterioridad en la posición de orientador. Sin embargo, con las sucesivas reformas educativas ha recalado como docente de las materias de Formación cívica y Ética, y Asignatura estatal dejando de lado su función de psicólogo-orientador. Asimismo, ejerce como profesor en una universidad local en la Facultad de Educación. El último de nuestros informantes es Juan, licenciado en Educación Media con la especialidad de Ciencias Sociales; imparte Formación cívica y Ética, en ambos turnos escolares. Su experiencia como docente en el instituto es de 13 años, de un total de 20 en otros centros educativos. Todos ellos han tenido experiencias como docentes en centros privados en sus inicios profesionales, aunque la mayoría de su trayectoria se ha desarrollado en la escuela pública: casi toda su vida laboral ha estado vinculada al centro que visitamos.

\section{RESULTADOS}

A continuación, repasamos algunos ejemplos en los que el lenguaje de las virtudes aparece de una manera, más o menos explícita, en la vida de estos docentes. En nuestro caso, no queremos realizar una descripción de cada una de las virtudes morales, intelectuales, etcétera, que expresan los docentes y sus correspondientes ejemplos. Centraremos nuestra atención en algunas virtudes visibles (Fallona, 2000) y ciertos elementos que los informantes destacan como importantes a la hora de promover las virtudes en la escuela.

\section{DOCENTES CON CORAJE}

Nos parece interesante destacar el coraje de los docentes, ya que culturalmente esta virtud, en el contexto mexicano, es vista como algo negativo, mayoritariamente como enojo. Sin embargo, al hablar del coraje lo definimos como la capacidad para actuar con valentía en situaciones difíciles (Klaassen, 2010); más en concreto, en el ámbito educativo, nos referimos a ella como la capacidad para emitir juicios en circunstancias 
difíciles sobre lo que se debe hacer y saber afrontarlo (Fallona, 2000). Aunque el coraje ha sido una de las virtudes sobre la que los docentes no centraban su atención en las entrevistas de una manera tan explícita como al hablar de otras, sí que a través de distintos acontecimientos, eventos y declaraciones hemos constatado de manera muy notable su presencia.

Pero, ¿por qué entendemos que estos docentes, principalmente Gabriel y Pedro, expresan la virtud del coraje en su actividad diaria? A continuación, expondremos algunos ejemplos que esperamos ayuden a comprender la personificación del coraje en los diferentes roles de estos docentes en la escuela. En primer lugar, nos gustaría destacar algunos elementos, como las pobres condiciones laborales, la diversidad sociocultural del alumnado, el exceso de estudiantes por aula, la escasez de apoyo interno (compañeros y parte del alumnado), externo (familias y administración educativa), los problemas sociales en el contexto del alumnado - ausencia parental, drogas, sexualidad, corrupción, violencia, acoso escolar, etcétera-, que afectan a los jóvenes mexicanos (García, Muñoz, y Orozco, 2007; INNE, n.d.) y que estos docentes deben superar diariamente. La cotidianidad de estas circunstancias y la interiorización de las mismas, permite que los propios docentes no sean conscientes del coraje moral que manifiestan en su escuela, y que les hace ejemplos de profesionalidad y compromiso con la docencia.

Así, siguiendo las categorías expresadas por Klaassen (2010), el coraje se puede expresar hacia los compañeros de trabajo, como responsabilidad pedagógica y autonomía docente. En este sentido, Gabriel lo manifiesta de dos maneras. Una de ellas luchando contra hábitos viciados en la escuela, en este caso la suciedad: «Muchos de los compañeros cuando están aquí en el salón, entro y me dejan un basurero, o sea, ellos permiten muchas veces basura» (Gabriel \#00:20:21-6); esto también es una realidad en algunos hogares "Comenté en una ocasión: "no sé, no creo que sean asi en su casa, que vivan con basura". Pues me creerás que sí..., porque hemos hecho visitas y han encontrado [basura]"»(Gabriel \#00:26:39-0\#). Ante esta situación, decidió tomar la iniciativa desarrollando un programa orientado a concienciar sobre la problemática, aunque lamentablemente no pudo lograrlo. "Hice también un proyecto de basura [...] pero no me ha funcionado, porque no tenemos cultura, [...]. Pedi ayuda a los 
tutores y no he tenido respuesta, son muy reacios para esto [...]. Hablé con 18 maestros, les pedi eso, eh a lo mejor alguno hizo, alguno sí, pero la mayoría no. Entonces yo me siento decepcionado porque era algo que cambiarle la mentalidad al alumno» (Gabriel \#00:22:37-6\#).

\section{Figura 1. Ejemplo de lona proyecto valores pagada por Gabriel}

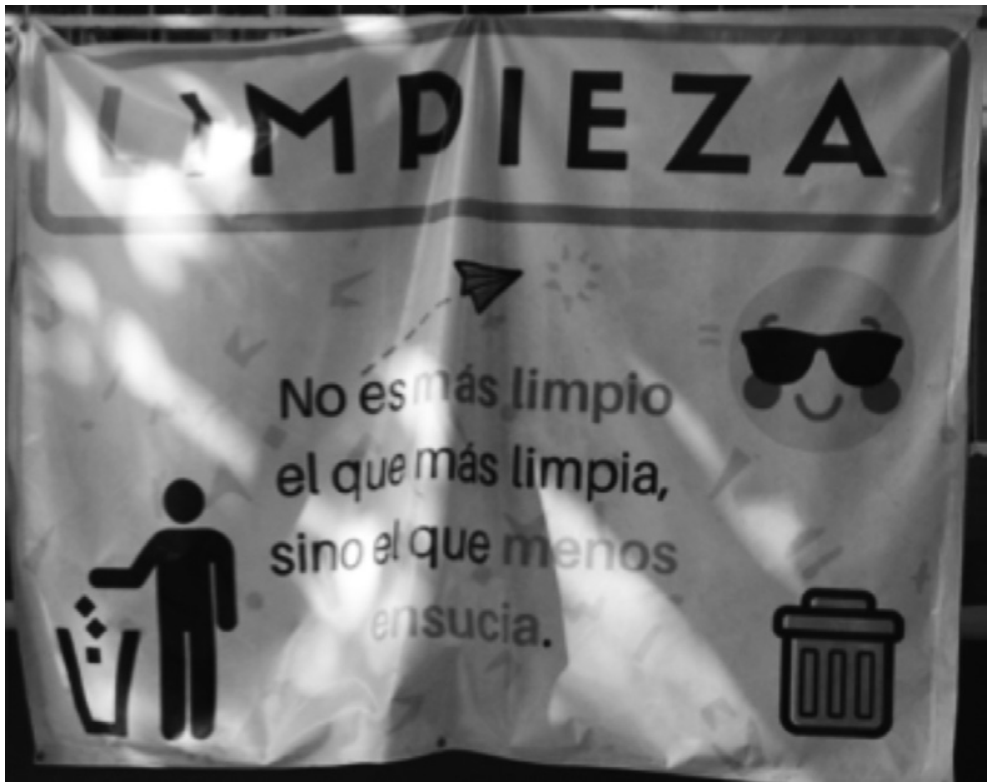

La segunda manera es involucrándose en iniciativas que ayuden a mejorar el clima escolar, como el proyecto de valores desarrollado en la escuela y que él apoya incluso aportando dinero propio para materiales, por propia convicción personal y siendo honesto con sus propios principios tanto como docente, compañero y ciudadano, "Ahorita se está llevando un proyecto de valores, [...] y entonces se ponen cartulinas, yo he regalado esas cartulinas que están ahi, yo me comprometí y cada mes doy de mi dinero [...]. Porque yo sí presumo de que tengo valores, sílo puedo presumir porque los conduzco en clase y los llevo a cabo en mi vida privada» (Gabriel \#00:12:31-8\#). 
Pedro también ha tenido que demostrar su coraje ante los boicots que ha sufrido por parte de algunos compañeros y familias. En este caso la problemática surge por algunos temas que propone en sus clases. «Te voy a poner un ejemplo, cuando se tocan temas de controversia, eh, si va a trabajar el tema de sexualidades y particularizando en aborto o de las cuestiones de paternidad, etcétera. Los papás [...] que no les parece, vienen y establecen como una oposición a decir que a ellos les corresponde esa parte formativa y otro grupo de padres dice que [...] nos corresponde a nosotros. Por eso están las generaciones, así como desordenadas» (Pedro \#00:23:00-4\#).

Como observamos, el conflicto surge por los distintos roles que adoptan estos agentes y por las responsabilidades que asumen. Mientras que el docente se preocupa por facilitar a todos los escolares información sobre temas próximos a sus problemáticas, algunas familias se preocupan legítimamente por lo que consideran mejor para sus hijas e hijos, algo común en muchas escuelas (Sanders, 2009).

Ante estas situaciones, Pedro procura tratar con mucha delicadeza los temas sin buscar un adoctrinamiento. Él mismo reflexiona al respecto, resaltando su idea de mantener un equilibrio entre el contenido moral y las formas morales (Fenstermacher et al., 2009): «Tengo mucho cuidado de estar como mediando entre [...] los valores que ellos ya tienen como referenciados en su contexto y lo que supuestamente se apegan a ello, o se apropian de ello para sostenerse en su convivencia» (Pedro \#00:18:37-7\#).

Por su propia experiencia personal y profesional, sabe que ciertos temas no son tratados en muchas escuelas mexicanas y bajo su prisma no responde a criterios pedagógicos, «Hay estados de la República, aqui en México, que cuando hablas de esos temas, por ejemplo, son cortadas las hojas de los libros de texto, otro estado quema todos los libros, [...] de repente quien gobierna te marca la pauta de la condición moral, no ética» (Pedro \#00:50:51-7\#).

Pedro nos explica que estos jóvenes no tienen capacidad para autorregularse; por esta razón, y a pesar de los incidentes sufridos y del esfuerzo que requiere, busca temas controvertidos, actuales y que afectan a los adolescentes, como la corrupción y su ejemplificación en el aula con los robos en las clases. «Estas cuestiones de la corrupción, incidir mucho en esa parte de que tienen que ser por lo menos honestos [...], te digo que te lleva tiempo en ir regulando, regulando. Tú les preguntas [...] qué 
opinan de los corruptos y te dicen la letanía completa, como si los odiaran en ese sentido, pero sus actos dicen otra cosa; entonces [...] me cuesta trabajo hacer la mediación» (Pedro \#00:22:10-8\#).

\section{TRATANDO DE MODELAR VIRTUDES}

Resulta interesante subrayar la honestidad que muestran los docentes al afirmar que de manera intencionada tratan de modelar determinadas virtudes en sus clases. Es curioso comprobar la coincidencia en algunas de estas virtudes. Los tres docentes centran su atención en virtudes morales, posiblemente como consecuencia de su experiencia en el centro, su capacidad para identificar las necesidades del alumnado y el contexto social en el que se relaciona, sus propias experiencias previas y los propósitos que tratan de alcanzar en sus clases; en definitiva, fruto de su sabiduría práctica en este contexto. Durante la estancia en sus clases, constatamos que a través de sus diferentes estilos y formas, son capaces de practicar el lenguaje de las virtudes de manera más o menos simbólica.

\section{Figura 2. Virtudes a modelar}

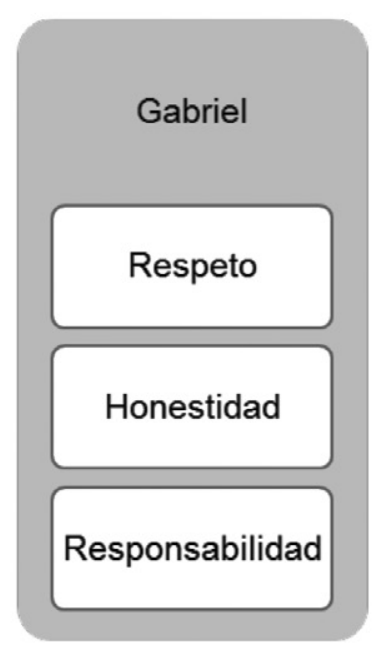

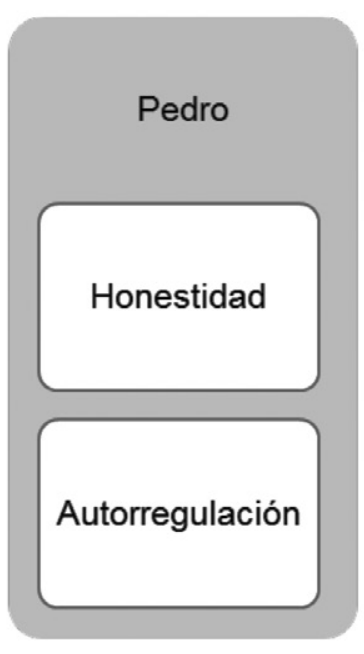

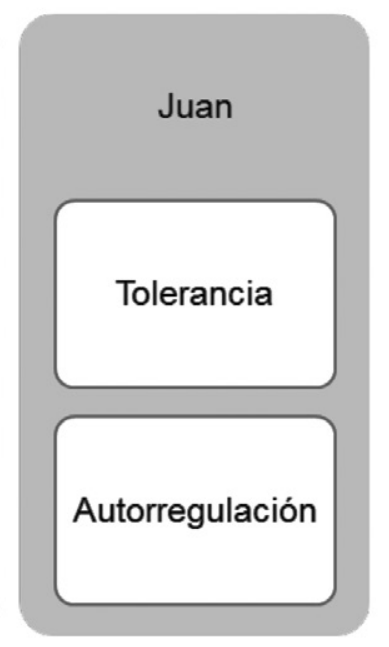

REVISTA PANAMERICANA DE PEDAGOGÍA n. 26 (2018): 167-187. SABERES Y QUEHACERES DEL PEDAGOGO 
Para lograr que los estudiantes adquieran estas virtudes es interesante subrayar que, para Gabriel, el tema de las rutinas-normas en el aula es fundamental para mantener un clima en el que se expresen las virtudes que trata de inculcar a su alumnado. Gabriel admite con naturalidad "Soy muy enérgico, tengo un carácter fuerte, así son las cosas, y Pedro me lo ha dicho a mi: "[...] Profe, los chavos luego no te quieren". Ah - digo-, yo no puedo cambiar mi carácter (Gabriel \#00:34:16-4\#). Quizás por eso mantiene un tono firme en sus clases, pero al mismo tiempo es capaz de ser jovial y bromear en el aula; recibe a los estudiantes en la puerta; revisa que todos lleguen puntuales, con el uniforme limpio; que no entren comiendo al aula, y saluda prácticamente a todos de manera individual. Es decir, se preocupa de que los escolares tengan claras las rutinas y las normas de juego, para que sepan lo que se demanda de ellos en clase. «Desde que entro a clase, llevo a cabo tres valores fundamentales: respeto, honestidad y responsabilidad. La responsabilidad primeramente porque ellos tienen que llegar temprano [...]. La honestidad me baso en ella porque cuando reviso los trabajos, les pido que me muestren el trabajo desde su lugar [...]. Después, aleatoriamente pregunto [...]. Lo que intento es que los muchachos sean honestos. El respeto para mi es fundamental tanto con mi familia, con mis amigos y sobre todo con los alumnos. Cuando participan ellos, normalmente les pido que digan siempre la verdad porque me va a molestar que me digan todo lo contrario; $y$ la otra es que cuando el alumno está participando, no permito que se rían de él, porque así le quiero dar la confianza para se exprese, manifieste sus ideas (Gabriel \#00:04:05-4\#).

Pedro en cambio, no establece unas rutinas-normas tan definidas. Los alumnos no tienen establecidos asientos fijos, los recibe en la zona del profesor y permite que se relajen hasta que él indica el comienzo; en ese intervalo, aprovecha para hablar con algún alumno o preparar materiales. Finalmente les saluda de manera general a todos en un tono suave con un "Buenos días», mantiene un tono bajo y casi nunca alza la voz para reclamar la atención de la clase. Desde su background, lo que busca es incidir en la autorregulación y el respeto en sus clases. «La incidencia es justamente sensibilizar por lo menos en el respeto de las ideas de los otros, el respeto de un estilo de vida en particular que ellos han elegido, y que obviamente que no afecte a terceros, que no sean estilos de vida radicales que pudieran alterar también las condiciones 
de posibilitar la convivencia [...] de relacionarse, cuando tienes que hacer algunos altos, algunas reflexiones, algunos intentos de autorregulación (Pedro \#00:14:19-2\#). A pesar de las diferencias en sus objetivos y en sus formas de enseñar, los dos generan un clima de cordialidad, confianza y respeto con los escolares desde el primer momento. Esto facilita la exposición de las virtudes en las clases, tanto de manera explícita como implícita.

Los docentes destacan la necesidad de promover modelos adecuados en la escuela. Ellos asumen, con naturalidad, que deben ser un ejemplo, eso sí cada uno con su método, estilo y forma, destacando que ser modelo no debe ser una cuestión sin importancia, debido a la complejidad que ello supone y al impacto que puede tener sobre los escolares. «No es que no quiera tomar esa responsabilidad, sino que el simple hecho de que sea su maestro, ya vas a dejar algo bueno o algo malo para ellos, porque [...] no a todos les vas a caer bien» (Juan 00:31:37-1\#). Ellos no se ven solamente como unos docentes, sino que dentro de la escuela deben jugar otros papeles para ayudar a su alumnado: «También el maestro tiene que hacer todas las acciones desde psicólogo, de sacerdote, de médico» (Gabriel \#00:27:03-8\#).

En sus declaraciones y actos, emerge la necesidad de transmitir un modelo ejemplar que permita a los escolares identificar las virtudes, para que puedan aprenderlas y llevarlas a la práctica en su vida diaria. "Les pongo siempre el ejemplo, si tú vas a la calle, cuando salgas de la secundaria, no vas a llevar tus calificaciones aqui en el pecho para que digan: "oye, tú eres un buen estudiante, sacaste puro 10". Pero sí, si te ven, que te levantas a cederle el asiento a la señora que está embarazada, a la anciana, ahí si te van a ver y van a decir, oye te fijaste el hijo de fulanita de tal, qué educado es; eso es lo que se ve» (Juan \#00:28:05-4\#).

Por esta razón como apunta Kristjánsson (2006) es frecuente el empleo, más o menos sistemático, de modelos ejemplares (historias, leyendas, biografías, etcétera) en el aula, que motiven a los estudiantes a emularlos: «Hoy les puse, por ejemplo, la frase de Martin Luther King que "hemos aprendido a volar como las aves..." (Juan, \#00:34:54-5\#). Pero la cuestión de los modelos no es solamente una cuestión de ser y de parecer un docente ejemplar hacia los alumnos, sino que también requiere que escolares y docentes lo demanden. 
Juan (menos experimentado), busca sus propias referencias en la escuela. Además, reconoce con humildad, que él también se inspira en algunos colegas para mejorar: «Yo, por ejemplo, admiro mucho a Fiorela ${ }^{2}$. Ella sí es un modelo a seguir, le digo: "tú eres mi máster, Fiorela". Es muy buena. Y la maestra Daniela también es muy buena, [al igual que] el maestro Rogelio» (Juan \#00:50:05-8\#). En este sentido, también parte de las chicas y los chicos demandan o exploran referentes que les ayuden a crecer desde el punto personal y social. «Mira, [...] obviamente se acercan con ídolos prefabricados, pero de repente si vas encontrando algunos niños que andan buscando un referente en términos de una persona que ha tenido como éxito a través de un esfuerzo, no el éxito nada más de repente, marcado como la estrellita que sale en Hollywood [...]. Inclusive tengo un trabajo por ahi de "quién es tu ídolo representativo, el más importante". [...] Ahora fijate algo interesante: parcialmente el papá ahora sale como el ídolo, no son pocos, andan como en la búsqueda no sé si de prototipos, no sé si referentes que pudieran ellos entenderlo como diferentes, porque no son los populares, se están sacando referentes, así como más, más en la búsqueda de la cualidad» (Pedro \#00:16:16-0\#).

No obstante, ellos mismos reconocen que la tarea de ser un ejemplo virtuoso no es fácil en el contexto sociocultural que viven los estudiantes. «Sin embargo, aquí en México, como que se enseña una doble moral. Por un lado, pedimos a los hijos que pongan en práctica esas virtudes, pero por otra parte con nuestro ejemplo, decimos lo contrario» (Juan 00:05:09-4\#).

Los docentes manifiestan su frustración al ver cómo fuera de sus clases, los escolares se ven expuestos a circunstancias muy duras (crimen, narcotráfico, violencia en el hogar, abandono familiar, etcétera) y que esto supone «gran conflicto» (Juan) en los escolares, ya que las realidades que viven en su día a día chocan con lo que ellos les proponen en sus interacciones en el aula y la escuela, dificultando gravemente la comprensión, interiorización y práctica de las virtudes en los adolescentes.

2 Hemos alterado los nombres para garantizar el anonimato y la confidencialidad de los docentes mencionados en la cita. 


\section{CONCLUSIONES}

En este estudio hemos explorado cómo se cristalizan determinadas virtudes en la actividad diaria de los docentes y cómo se tratan de promover en un instituto público emergente de educación secundaria. En primer lugar, constatamos la importancia de la reflexión del docente en torno a su compromiso moral, ético y pedagógico con el contexto sociocultural que le rodea, y cómo a partir de esta concienciación, la expresión de las virtudes puede desarrollarse en la práctica diaria, con mayor o menor intensidad.

Es conveniente resaltar que en contextos de gran diversidad cultural, el coraje moral docente resulta fundamental para afrontar la compleja tarea de educar en virtudes, resolviendo las tensiones entre lo que demanda el currículo, los compañeros, las familias, los adolescentes, sus propósitos y la autonomía como docente.

Por otra parte, destacar la necesidad de reflexionar sobre la propia experiencia personal y de cómo ésta se relaciona con la sabiduría práctica de los docentes a la hora proyectar un estilo y obtener formas apropiadas a la hora de presentar contenidos, establecer rutinas, etcétera, que permitan manejar el lenguaje, más o menos oculto, de las virtudes en sus contextos educativos.

Es evidente la importancia que juega el rol-model para transmitir las virtudes, pero no menos relevante son los roles que los docentes manejan en función de su posicionamiento ante el alumnado, el compañero o hacia uno mismo en el momento de suscitar virtudes.

El docente no es solamente modelo o presentador de modelos inspiradores, sino que también ha de ser un observador demandante de ejemplos que inspiren su aprendizaje, hasta llegar a ser un docente virtuoso, favoreciendo así un impacto positivo en los estudiantes.

Por último, es importante destacar la soledad y frustración que debe superar el docente cuando el contexto escolar y sociocultural no comparte, o posee, los principios éticos, morales y profesionales necesarios para la tarea de ayudar a los jóvenes en su florecimiento personal y social. 


\section{REFERENCIAS}

Altarejos, F., y Naval, C. (2000). Filosofía de la educación. Pamplona: Eunsa.

Angrosino, M. (2012). Etnografía y observación participante en investigación cualitativa (1 $\left.{ }^{\mathrm{a}}\right)$. Madrid: Morata.

Arthur, J., \& Kristjánsson, K. (2015). Is Good Character Caught or Taught. Retrieved from https://www.bigquestionsonline. com/2015/02/17/good-character-caught-taught/

Arthur, J., Kristjánsson, K., Harrison, T., Sanderse, W., \& Wright, D. (2017). Teaching Character and Virtue in Schools. London-New York: Routledge.

Bernal, A. (1998). Educación del carácter, educación moral: propuestas educativas de Aristóteles y Rousseau. Pamplona: Eunsa.

Bernal, A., González-Torres, M. C., y Naval, C. (2015). La Educación del carácter perspectivas internacionales, (4), 35-46. Retrieved from https://sede.educacion.gob.es/publiventa/ la-educacion-del-caracter-perspectivas-internacionales / educacion-politica-educativa/ 20413

Biesta, G. J. J. (2014). How does a competent teacher become a good teacher? On judgement, wisdom and virtuosity in teaching and teacher education. In R. Helilbronn \& L. Foreman-Peck (Eds.), Philosophical perspectives on the future of teacher education (pp. 3-22). Oxford: Whiley Blackwell. https://doi. org/10.1002/9781118977859.ch1

Bolívar, A. (1987). Desarrollo moral y educación moral: la perspectiva cognitivo-formalista. Revista Española de Pedagogía, XLV (177), 395-409. Retrieved from https://revistadepedagogia. org / wp-content / uploads / 2018 / 01 / Evolución-y-perspectivas-de-la-educación-moral-1.pdf 
Campell, E. (2013). The virtuous, wise, and knowledgeable teacher: living the good life as a professional practitioner. Educational Theory, 63(4), 413-429. https://doi.org/https://doi. org / 10.1111/ edth.12031

Carbajal, P. (2009). Una aproximación a la comprensión de la conciencia moral de docentes de Educación Básica a través de un proyecto de intervención, 7, 162-183. Retrieved from https:/ / revistas.uam.es / index.php/ reice/article/view / 5417

Carrillo, I. (1992). Análisis crítico del entorno, elección de alternativas y clarificación de valores. Comunicación, Lenguaje y Educación, (15), 77-83. Retrieved from file:///C:/Users/agrana/ Desktop/Character Education Project Novo/Character Biblio/ Dialnet-AnalisisCriticoDelEntornoEleccionDeAlternativasYCl-126262.pdf

Devries, R. (1999). Implications of Piaget's Constructivist Theory for Character Education. Action in Teacher Education, 4(20), 39-47.

Fallona, C. (2000). Manner in teaching: a study in observing and interpreting teachers and moral virtues. Teaching and Teacher Education, 16, 681-695. https://doi.org/10.1016/S0742051X(00)00019-6

Fenstermacher, G. D. (1992). The concept of Method and Manner in Teaching. In F. K. Oser, A. Dick, y J.-L. Patry (Eds.), Effective and Responsible Teaching (pp. 95-108). San Francisco: Jossey-Bass Publishers.

Fenstermacher, G. D., Osguthorpe, R. D., \& Sanger, M. N. (2009). Teaching Morally and Teaching Morality. Teacher Education, 36(3), 7-19. Retrieved from https: / / files.eric.ed.gov/fulltext/ EJ858720. pdf

Flick, U. (2004). Introducción a la investigación cualitativa. Retrieved from https://books.google.com/books?id=o0iLN8Ag8ewCypgis $=1$ 
Gordillo, M. V. (1987). El desarrollo moral de la mujer. Revista Española de Pedagogía, LXX (253), 29-43. Retrieved from https: / / revistadepedagogia.org/wp-content/uploads / 2018/01/Evolución-y-perspectivas-de-la-educación-moral-1.pdf

Jackson, P. W. (1991). La vida en las aulas. Madrid: Morata.

Klaassen, C. A. (2010). Teachers' Views and the Need of Moral Courage. In C. A. Klaassen y N. Maslovaty (Eds.), Moral Courage and the Normative Professionalism of Teachers (pp. 13-26). Rotterdam-Boston-Taipei: Sense Publishers.

Kristjánsson, K. (2006). Emulation and the use of role models in moral education. Journal of Moral Education, 35(1), 37-49. https: / / doi.org/10.1080/03057240500495278

Lozano, N. (2015). Education for Peace in the Mexican context. Canadian Journal of Latin American and Caribbean Studies, 3663(June), 37-41. https: / / doi.org/10.1080/08263663.2015.1044720

Martino, W. (2009). Teacher as Role Models. In L. J. Saha y A. G. Dworkin (Eds.), International Handbook of Research on Teachers and Teaching (pp. 755-768). Boston, MA: Springer. https:// doi.org/ https: / / doi.org/10.1007/978-0-387-73317-3_47

Miles, M., Huberman, A., \& Saldaña, J. (2014). Designing matrix and network displays. In Qualitative Data Analysis. A Methods Sourcebook (nd, pp. 107-119). Thousand Oak, California: Sage Publications.

Milner, H. R. (2015). Research on Classroom Management in Urban Schools. In E. T. Emmer y E. J. Sabornie (Eds.), Handbook of Classroom Management (pp. 167-185). New York-London: Routledge.

Moulin-Stozek, D. (2018). The Social Construction of Character. Social Theory, (aceptado, pendiente de publicación).

Moulin-Stozek, D., \& Schirr, B. J. (2017). Identification and disidentification in reported schooling experiences of adolescents 
Muslims in England. Oxford Review of Education, 43(5), 580-595. https: / / doi.org/https: / / doi.org/10.1080/03054985.2017.1352353

Naval, Fuentes, J. L., y Quintanilla, I. (2017). La educación para una ciudadanía global. Buenas teorías que orientan buenas prácticas. En M. Á. Hernández (Ed.), Educación para la vida ciudadana en una sociedad plural (pp. 243-272). Murcia: Editum.

Noddings, N. (2008). Caring and Moral Education. In L. P. Nucci y D. Narváez (Eds.), Handbook of moral and character education (161-174). New York: Routledge.

Noddings, N. (n.d.). Caring in Education. Retrieved from http:/ / infed.org/mobi/ caring-in-education/

Noddings, N. (1984). Caring, a feminine approach to ethics y moral education. Berkeley-Los Angeles-London: University of California Press.

Piaget, J. (1984). El criterio moral en el niño. Barcelona: Ediciones Martínez Roca.

Richardson, V., \& Fallona, C. (2001). Classroom management as method and manner. Journal of Curriculum Studies, 33(6), 705-728. https: / / doi.org/10.1080/00220270110053368

Sanders, M. G. (2009). Teachers and Parents. In International Handbook of Research on Teachers and Teaching (pp. 331-341). New York: Springer International Handbooks of Education 21.

Sanderse, W. (2013). The meaning of role modelling in moral and character education. Journal of Moral Education, 42(1), 28-42. https: / / doi.org/10.1080/03057240.2012.690727

Simons, H. (2011). El estudio de caso: teoría y práctica. Madrid: Morata.

Spradley, J. P. (1979). The ethnographic Interview. Belmont: Wadsworth Cengage Learning. 
Stake, R. E. (1998). Investigación con estudio de caso (2a Ed). Madrid: Morata.

Taylor, S. J., y Bogdan, R. (1992). Introducción a los métodos cualitativos de investigación. Barcelona: Paidós Básica.

The Jubilee Centre for Character \& Virtues. (2017). A framework for Character Education in Schools. Birmingham: The Jubilee Center for Character y Virtues. Retrieved from https://uobschool. org.uk/wp-content/ uploads / 2017 / 08 / Framework-for-Character-Education-2017-Jubilee-Centre.pdf

Willemse, M., Lunenberg, M., \& Korthagen, F. (2008). The moral aspects of teacher educators' practices. Journal of Moral Education, 37(4), 445-466. https: / / doi.org/ 10.1080/03057240802399269

Yurén, T., Saenger, C., y Rojas, A. (2014). Prácticas de investigación sobre formación moral en México: Meta-análisis de un estado del conocimiento. Actualidades Investigativas en Educación, 14(1), 1-22. Retrieved from http://www.redalyc.org/ pdf/ 447/44729876010.pdf 